Journal of Machine Engineering, 2021, Vol. 21, No. 3, 40-46

ISSN 1895-7595 (Print) ISSN 2391-8071 (Online)

Received: 25 June 2021 / Accepted: 24 August 2021 / Published online: 28 August 2021

machine tool, cloud computing, Industry 4.0, edge computing

Jürgen FLEISCHER ${ }^{1 *}$

Alexander PUCHTA ${ }^{1}$

Philipp GÖNNHEIMER ${ }^{1}$

\title{
SEAMLESS AND MODULAR ARCHITECTURE FOR AUTONOMOUS MACHINE TOOLS
}

\begin{abstract}
In machine tools, existing solutions for process monitoring and condition monitoring rely on additional sensors or the machine control system as data sources. For a higher level of autonomy, it becomes necessary to combine several data sources, which may be within or outside of the machine. Another requirement for autonomy is additional computing power, which may be hosted on edge devices or in the cloud. A seamless and modular architecture, where sensors are integrated in smart machine components or smart sensors, which are in turn connected to edge devices and cloud platforms, provides a good basis for the incremental realisation of autonomy in all phases of the machine life cycle.
\end{abstract}

\section{INTRODUCTION}

The goal of autonomous machine tools is to increase overall equipment effectiveness (OEE) and achieve lower operating costs. To achieve this goal, Industry 4.0 solutions for machine tools are being developed in the areas of condition monitoring, predictive maintenance and process monitoring. Condition monitoring and predictive maintenance solutions increase availability and reduce maintenance costs. Process monitoring serves to increase quality and productivity.

This is becoming more and more relevant as highly variable products lead to ever smaller batch sizes. In addition, the demands on productivity and product quality are increasing. Volatile markets also require more flexibility in production. In parallel, the availability of solutions for data acquisition and processing is increasing.

However, the solutions are not yet being used across the board due to a diverse hardware and software landscape and the fact that most applications are only implemented as isolated applications. Thus, there is no universally applicable solution. In order to implement Industry 4.0 across the board, a seamless and modular architecture is needed to integrate new sensors [1].

\footnotetext{
${ }^{1}$ wbk Institute of Production Science, Karlsruhe Institute of Technology (KIT), Germany

*E-Mail: juergen.fleischer@kit.edu

https://doi.org/10.36897/jme/141565
} 


\section{VISION AUTONOMOUS MACHINE TOOLS}

To achieve autonomy of machine tools, the entire life cycle of a machine must be considered. For this purpose, Industry 4.0 functions were collected in the state of the art and research. These were sorted according to topics. This resulted in 5 clusters in a life cycle of a machine tool. The five areas are: Developing, Machine production \& Commissioning, Operation, Maintenance and Reuse. Figure 1 shows the different phases and the associated tasks that ensure the highest possible level of autonomy in each phase. In the development phase, machine tools can model itself and make use of data-driven simulations. In addition, new kinematics can increase the degree of autonomy. In Machine production \& commissioning, the machine can describe and configure itself. In addition, quality checks are performed autonomously. During the operation of the machine, self-optimizations take place to increase the OEE. The different components communicate decentrally. In addition to selfoptimization, systems can also be used to support the operator and make suggestions for machine settings. In contrast to this is the user-free operation in which the machine settings are set directly by an algorithm. An important aspect in the operation of machine tools is maintenance. Here, the machine monitors individual components itself and can diagnose the current condition itself. In addition, the machine can predict when the monitored component will fail and initiate the maintenance steps itself. In order to increase the autonomy of existing machines, retrofit takes place in the reusage phase. Here, the machine is equipped with additional sensors and computing units to cover the points already mentioned in Operation and Maintenance. In the case of an autonomous machine, self-decommissioning is at the end of the life cycle.

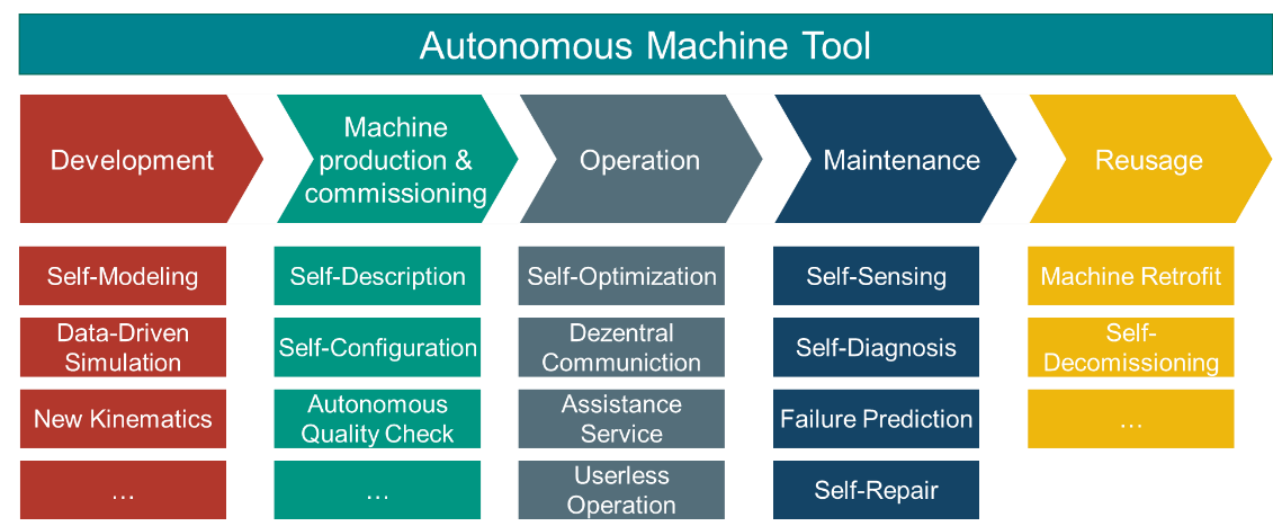

Fig. 1. Lifecycle of autonomous machine tools

\section{REALISATION OF AUTONOMOUS MACHINE TOOLS}

Such a high degree of autonomy presupposes that additional functions can be added at any time for the characteristics described above, especially in the areas of operation, maintenance and reusage. 
In many cases, the additional functions use data from the control system. In process monitoring, for example, anomalies can be detected using signals that have already been recorded [2]. However, many applications require additional sensors. For example, pittings on ball screws can be detected using cameras [3]. Alternatively, the condition of a ball screw can also be predicted using vibrations $[4,5]$ or strain $[6,7]$. Furthermore, the service life of a ball screw can be extended by adaptive lubrication [8].

Based on the additional functions presented in the state of the art and research, the following requirements arise for the architecture of the system. On the one hand, additional computing power is required to realise the functions. On the other hand, the possibility of integrating additional sensors or intelligent components into the architecture is required. The system must have a modular structure in order to integrate the individual functions depending on the use case.

In addition, the Industry 4.0 functionalities shown also require control data, which is not available in a uniform way for heterogeneous machine parks. Therefore, an additional tool is needed to identify the necessary parameters [9].

\section{SEAMLESS AND MODULAR ARCHITECTURE}

In order to seamlessly and modularly equip any machine tool with Industry 4.0 functions, all data sources of the machine (control data, smart components, smart sensors) must be made available to the additional computing unit.

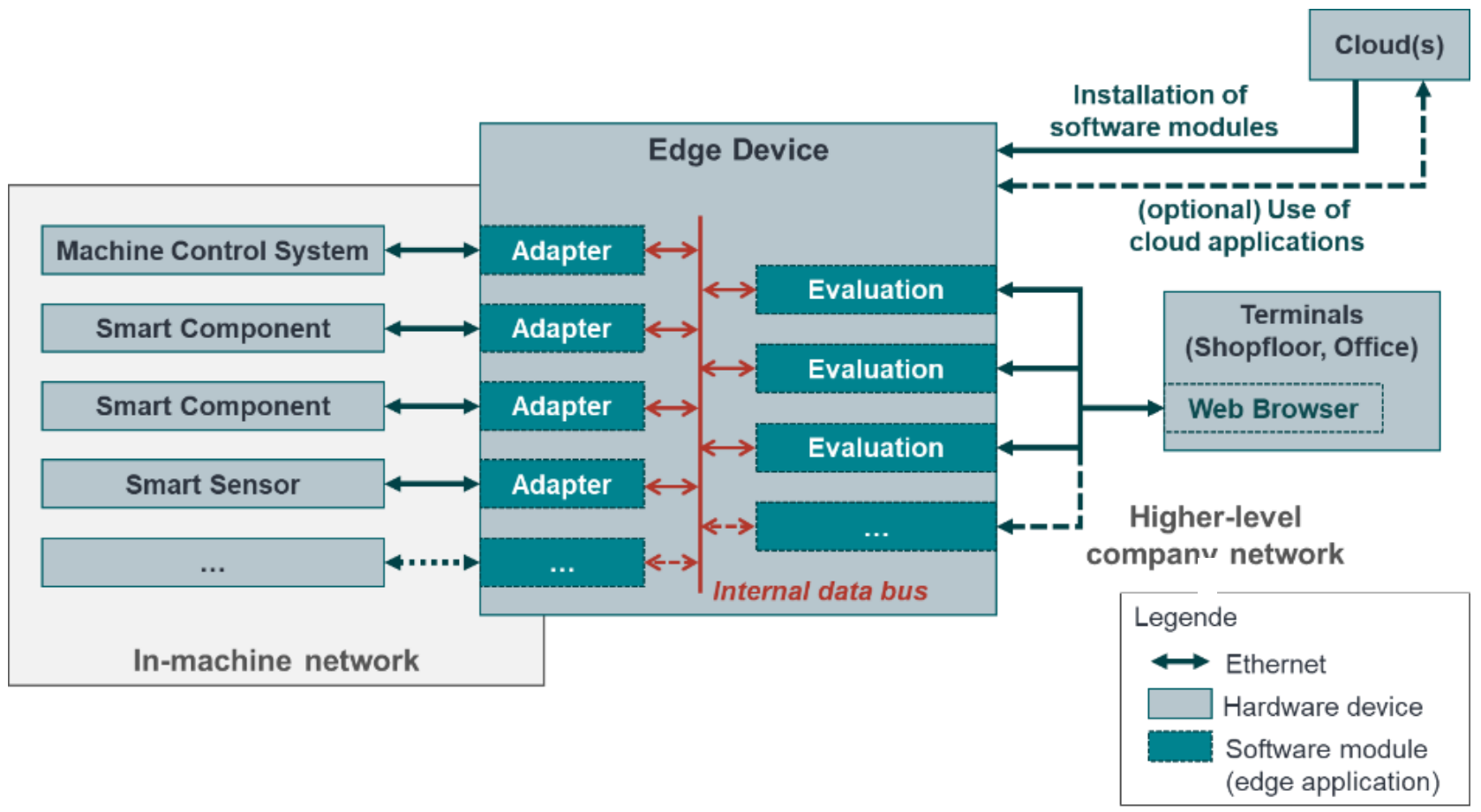

Fig. 2. Seamless and modular architecture [1]

For this purpose, all participants are integrated into the local machine network. The additional computing unit for data processing and visualisation of the various 
applications is an edge device. Depending on which Industry 4.0 applications are required by the machine operator, the necessary smart components and smart sensors are integrated into the machine network via Ethernet and the necessary adapters are installed on the edge device.

The necessary software modules are installed via the cloud. The applications installed in this way make the calculated data available to the higher-level company network. Optionally, cloud applications can be integrated if the computing power of the Edge is not sufficient and/or no time-critical reaction is required, e.g. cross-machine dashboards. The endto-end and modular architecture is shown in Fig. 2. This architecture was used to retrofit an existing machine [1].

Through the cloud connection, updates and new applications can be distributed centrally to many machines within a very short time. Thus, the presented architecture can be rolled out to any machines with individual configurations of Industry 4.0 functionalities. For example, chatter can be detected by introducing an acceleration sensor close to the tool centre point and the data from the control system [11]. In addition, further applications such as tool condition monitoring and force monitoring have been developed, which only require data from the control system. All applications can be used for new and old machines.

\section{EDGE DEVICE OR CLOUD}

The architecture uses a combination of edge device and cloud. The integration of more sensors into the architecture for the different applications leads to larger data streams. In a centralised network architecture, this would lead to overload. The nodes of the network, i.e. sensors and actuators, thus receive and/or send too much data. These can amount to several $\mathrm{GB} / \mathrm{s}$ under certain circumstances. Direct transmission of all data to a central platform is therefore not possible. Therefore, an edge device is used in the architecture. This is connected locally to a system, i.e. to a group of sensors/actuators. On the other hand, the edge device is connected to the cloud in order to be managed as already mentioned and also to have access to further computing power. Through an edge device, data is processed locally and only selected data is transmitted in aggregated form to the cloud or to the user. In addition, data security can be increased with an edge device. In order to be able to use the additional computing power sensibly, it must be assessed for each application to what extent it should be executed at the edge or in the cloud. This depends on the following factors [1]:

- IT security.

- Data protection and data sovereignty.

- Data volume and bandwidth.

- Computing capacity.

- Latency.

For example, the response time of an application to detect tool wear is only a few seconds and must therefore be evaluated on an edge device. In contrast, the reaction time for wear detection of a ball screw is several hours and can therefore be outsourced to the cloud. It is advisable to outsource as much as possible to the cloud, as this has greater computing power and relieves the edge. Due to the decreasing latencies in data transmission, the cloud will move more into the foreground in the future. 


\section{SMART CONTROLLER}

As already mentioned, Industry 4.0 functionalities cannot be rolled out across a production area due to a heterogeneous machine field. With modular smart controllers, individual Industry 4.0 functions can be realised independently of the manufacturer and easily integrated into the seamless and modular architecture. In order to realise different applications, the smart controller must have different capabilities. Depending on the application, a real-time partition and a best-effort partition are necessary. For example, a real-time capability system is needed for collision detection and a best-effort partion for lifetime prediction. To be able to guarantee both partitions, a hypervisor is used. The software architecture of the two portions is shown in Fig. 4 [10].

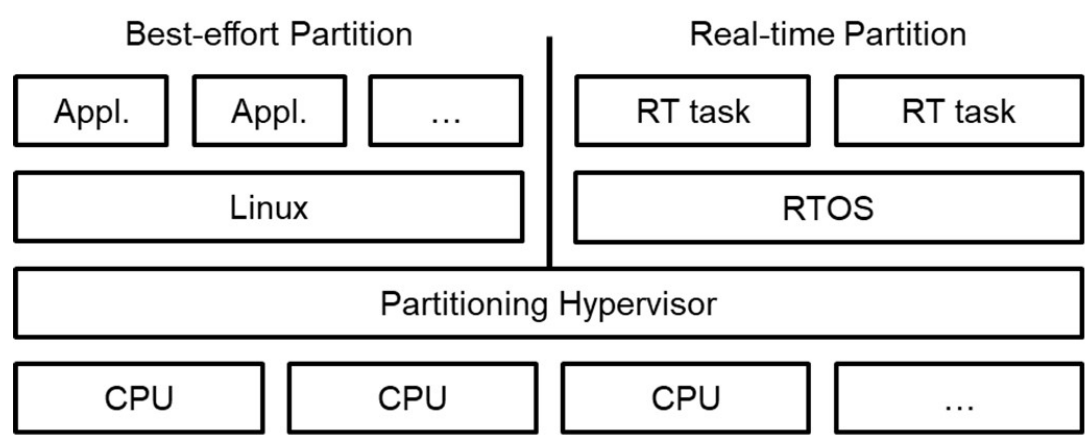

Fig. 3. Smart Controller software architecture [10]

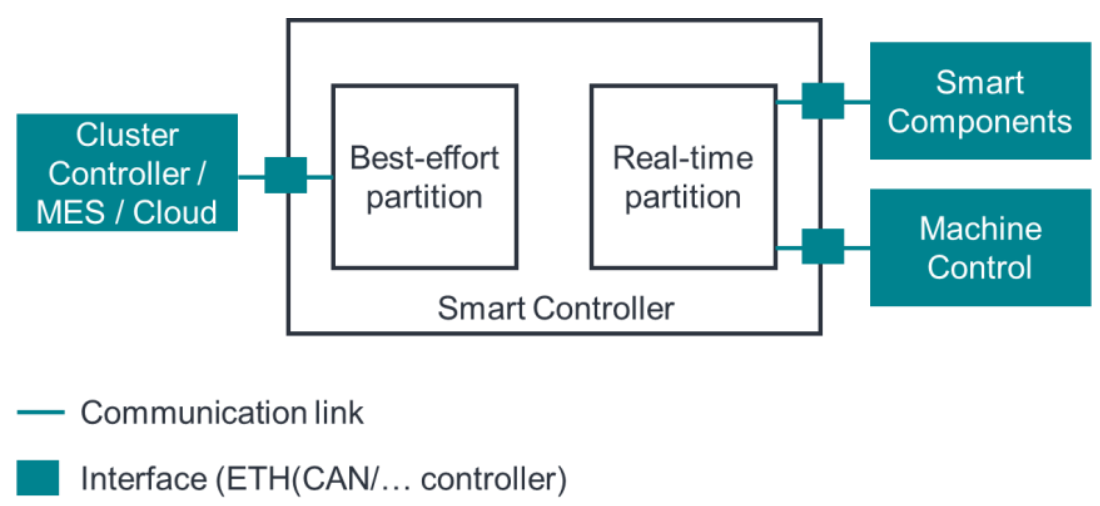

Fig. 4. Communication interfaces of the Smart Controller [10]

However, only a soft real-time capability is provided by the partitions running in parallel. This is sufficient for the smart controller, however, as the safety-critical functionalities are implemented directly in the machine controller. The best-effort partition has an interface to the higher management levels, such as a cloud. The real-time partition has the task of taking over Industry 4.0 functionalities for predictive maintenance and process monitoring. For these tasks, the partition has the interfaces to the machine control and the smart components. The concept of the smart controller is shown in Fig. 4. The smart controller consists of a Banana Pi [10]. 


\section{IDENTIFICATION OF CONTROL SYSTEM PARAMETERS}

In order to provide the Industry 4.0 applications with the required data sources, the correct parameters must be recognised as mentioned above. For this purpose, a strategy was developed which assigns the parameters over several stages. Figure 5 shows the identifycation process. The input of the identification is represented by different signals of the control of machine tools [9].



Fig. 5. Parameter identification procedure [9]

In the first stage, these signals are classified into main groups using machine learning approaches such as random forests or convolutional neural networks. Subsequently, the second stage divides the signals of the main groups into further subgroups using correlation, mean values and maximum/minimum positions. In the last stage, the signals are assigned to the same axes on the basis of the correlations. Thus, the three-stage strategy draws on domain knowledge and machine learning approaches to identify the parameters [9].

The knowledge about the identified parameters can be passed on to the various Industry 4.0 applications in the seamless and modular architecture directly or via a shared memory. In this way, applications such as the above-mentioned anomaly detection can be integrated without great effort. Otherwise, a time-consuming manual assignment would be necessary, which in turn would conflict with rolling out the architecture to entire machine parks. By extending the existing parameter list, additional functions can be connected to the required data.

\section{CONCLUSION}

For the use of Industry 4.0 applications, additional sensors are often required, and a seamless and modular architecture is needed to integrate these with little effort. An edge device that is connected to a cloud is suitable for this. With the help of autonomous parameter 
identification, the integration of Industry 4.0 applications can also be facilitated. In addition, smart components can be integrated into the machine network via smart controllers or directly pass on their information to higher levels.

In future work, further functionalities for the retrofit kit architecture will be developed, which can be applied not only to metal cutting production. However, due to the lower latencies in data transmission, the focus will move from the edge to the cloud in the future. In addition, parameter identification and edge architecture must be brought together to form a manufacturer-independent platform in which Industry 4.0 can be integrated.

\section{REFERENCES}

[1] BARTON D., STAMM R., MERGLER S., BARDENHAGEN C., FLEISCHER J,. 2020, Industrie-4.0-Nachrüstkit für Werkzeugmaschinen: Modulare Lösung für zustandsorientierte Instandhaltung und Prozessüberwachung, wt Werkstattstechnik online, 110/7-8, 491.

[2] NETZER M., MICHELBERGER J., FLEISCHER J., 2019, Intelligente Störungserkennung einer Werkzeugmaschine, Zeitschrift für wirtschaftlichen Fabrikbetrieb, 114/10, 635-638.

[3] SCHLAGENHAUF T., FEURING C.P., HILLENBRAND J., FLEISCHER J., 2019, Camera Based Ball Screw Spindle Defect Classification System, Production at the leading edge of technology, Springer Vieweg, Berlin, Heidelberg, 503-512.

[4] ZHANG L., GAO H., et al., 2017, A Deep Learning-Based Recognition Method for Degradation Monitoring of Ball Screw with Multi-Sensor Data Fusion, Microelectronics Reliability, 75, 215-222.

[5] ELFORJANI M., SHANBR S., 2017. Prognosis of Bearing Acoustic Emission Signals Using Supervised Machine Learning, IEEE Transactions on industrial electronics, 65/7, 5864-5871.

[6] HILLENBRAND J., SPOHRER A., FLEISCHER J., 2018., Zustandsüberwachung bei Kugelgewindetrieben, wt Werkstatttechnik, online, 8, 493-498.

[7] MÖHRING H.C., BERTRAM O., 2012, Integrated Autonomous Monitoring of Ball Screw Drives, CIRP Annals, 61/1, 355-358.

[8] SPOHRER A., 2019, Steigerung der Ressourceneffizienz und Verfügbarkeit von Kugelgewindetrieben Durch Adaptive Schmierung, Shaker Verlag.

[9] GÖNNHEIMER P., PUCHTA A., FLEISCHER J., 2020, Automated Identification of Parameters in Control Systems of Machine Tools, Congress of the German Academic Association for Production Technology, Springer, Berlin, Heidelberg, 568-577.

[10] BARTON D., GÖNNHEIMER P., SCHADE F., EHRMANN C., BECKER J., FLEISCHER J., 2019, Modular Smart Controller for Industry 4.0 Functions in Machine Tools, Procedia CIRP, 81, 1331-1336.

[11] BARTON D., FEDERHEN J., FLEISCHER J., 2021, Retrofittable Vibration-Based Monitoring of Milling Processes Using Wavelet Packet Transform, Procedia CIRP, 96, 353-358. 